\title{
Construcción y aplicación de una herramienta metodológica para la estimación de amenazas, vulnerabilidad y la capacidad de adaptación a eventos climáticos extremos y cambio climático
}

\section{Construction and application of a methodological tool for the estimation of threats, vulnerability and the capacity to adapt to extreme climate events and climate change}

\author{
William Klinger Brahan', Lady Vargas Porras²
}

\section{Resumen}

Se diseñó una metodología para estimar las amenazas, el grado de vulnerabilidad y la capacidad de adaptación al cambio climático. Se construyó y aplicó como base para la formulación del Plan Integral de Cambio Climático del departamento del Chocó y se realizó con el objeto de generar información diagnóstica sobre los riesgos asociados con los fenómenos climáticos en el territorio y las condiciones que contribuyen a incrementar sus impactos en la población, los ecosistemas y las actividades productivas. Para el diseño de la metodología se levantó información acerca de la ocurrencia de eventos climáticos extremos y se tuvo en cuenta las líneas estratégicas de la política nacional de cambio climático; además, fue necesario dividir el territorio en subregiones de acuerdo con sus características ambientales para facilitar el análisis de la información por tipo de evento, línea estratégica y área geográfica. Se presentan los resultados de la aplicación de la metodología diseñada para la amenaza más frecuente en la región que corresponde a las inundaciones, obteniendo los mayores grados para las subregiones de Baudó (67\%) y Darién (77\%), las cuales coincidieron a su vez con presentar los mayores porcentajes de vulnerabilidad y riesgo frente a este evento climático, siendo más crítico el caso de la región Baudó. La herramienta permite que con el levantamiento de datos históricos climáticos e información ambiental y social diagnóstica, se pueda aplicar a cualquier zona del país, generando resultados para el análisis y priorización de estrategias de prevención de impactos frente al cambio climático.

Palabras clave: Amenazas, Cambio climático, Cálculo de capacidad de adaptación, Determinación de vulnerabilidad y riesgo, Eventos climáticos extremos.
Ingeniero Forestal, MSc. en Educación, Director del Instituto de investigaciones Ambientales del Pacífico (IIAP), Quibdó, Colombia.

e-mail: wklinger@iiap.org.co

IngenieraAmbiental y Sanitaria, MSc. (C) en Ciencias Ambientales, Investigadora Principal Componente Ambiental, Instituto de investigaciones Ambientales del Pacífico (IIAP), Quibdó, Colombia. e-mail: 1vargas@iiap.org.co 


\section{Bioetnia Volumen 12, 2015}

\section{Abstract}

A methodology was designed to estimate threats, vulnerability and adaptability to climate change. It was built and applied as a basis for the formulation of the Comprehensive Climate Change Plan of the Department of Chocó and was carried out in order to generate diagnostic information on the risks associated with climatic phenomena in the territory and the conditions that contribute to increase its impacts on the population, ecosystems and productive activities. For the design of the methodology, information was collected on the occurrence of extreme climatic events and the strategic lines of the national climate change policy were taken into account. Additionally, it was necessary to divide the territory into subregions according to their environmental characteristics to facilitate the analysis of information by type of event, strategic line and geographical area. The results of the application of the methodology designed for the most frequent threat in the region corresponding to floods are presented, obtaining the highest grades for the subregions of Baudo (67\%) and Darién (77\%), which coincided in turn with the highest percentages of vulnerability and risk in relation to this climatic event, being more critical the case of the Baudo region. The tool allows the survey of climatic historical data and diagnostic and social environmental information to be applied to any area of the country, generating results for the analysis and prioritization of strategies to prevent impacts against climate change.

Keywords: Adaptive capacity calculations, Climate change, Extreme weather events, Threats, Vulnerability and menace assessment.

\section{Introducción}

Se entiende por cambio climático un cambio de clima atribuido directa o indirectamente a la actividad humana que altera la composición de la atmósfera mundial y que se suma a la variabilidad natural del clima observada durante períodos comparables"; estas alteraciones en la atmosfera son causadas por el incremento de las emisiones que se entienden como la liberación de gases de efecto invernadero o sus precursores en la atmósfera en un área y un tiempo especificados. Estos gases son aquellos componentes de la atmósfera, tanto naturales como antropogénicos, que absorben y emiten radiación infrarroja (Naciones Unidas, 1992).

Por su parte, la variabilidad climática asociada con los fenómenos El Niño y La Niña regulan el número de tormentas, de granizadas, de incendios de vegetación o de crecientes o inundaciones súbitas o son las fases responsables de que en una región se presenten sequía o inundaciones prolongadas (CORTOLIMA-UNAL, 2015). Colombia es afectada constantemente por eventos hidrometeorológicos extremos en gran parte de su territorio: las inundaciones, deslizamientos, granizadas y olas de calor han incrementado su frecuencia en los últimos años, afectando las actividades económicas y a las personas de las zonas más sensibles (IDEAM, 2002). Muchos centros poblados del país están altamente expuestos a los impactos del cambio climático pues se encuentran ubicados en zonas de riesgo. La pobreza alcanza $64 \%$ del total de la población (PNUD, 2008), lo cual hace que el país presente una alta sensibilidad a los efectos del cambio climático (Gutiérrez y Espinosa, 2010).

Los fenómenos extremos del clima pueden contribuir a la ocurrencia de desastres; los riesgos de desastre surgen de la interacción entre fenómenos meteorológicos o climáticos extremos, coadyuvantes físicos junto con la exposición y vulnerabilidad, coadyuvantes desde el punto de vista humano (IPCC, 2012). Por tal motivo, cuando se habla de riesgo, depende del tipo de amenaza, el nivel de exposición y las condiciones de vulnerabilidad. Para medir el riesgo se deben identificar cuáles son las amenazas y sus 
efectos sobre los sistemas socioeconómicos y los ecosistemas, determinar el grado de exposición analizando los lugares donde se encuentran estos sistemas y finalmente, determinar los factores que componen la vulnerabilidad, es decir, aquellos que determinan la susceptibilidad o predisposición de que un sistema se vea afectado de forma negativa ante una amenaza. Cuando una amenaza se materializa en un evento, el riesgo se convierte en un desastre que se traduce en impactos socioeconómicos (PNACC, 2015).

Finalmente, según la OMM, IPCC y PNUMA (2008) la adaptación al cambio climático se refiere a los ajustes en sistemas humanos o naturales como respuesta a estímulos climáticos proyectados o reales, o sus efectos, que pueden moderar el daño o aprovechar sus aspectos beneficiosos; sin embargo esta adaptación parte del diseño de estrategias que permitan a las comunidades reaccionar de manera adecuada frente a los cambios en las condiciones climáticas y ambientales del medio y en este sentido esta construcción debe partir del entendimiento de la dinámica de los componentes socioambientales y de un diagnóstico que recoja todos los elementos que intervienen en la misma. Es así como se hacen necesarias metodologías que permitan estimar las amenazas, el grado de vulnerabilidad, el riesgo y la capacidad de adaptación de las comunidades frente a eventos climáticos, cuya aplicación y resultados sean útiles a la hora de generar programas preventivos y correctivos de las impactos que se puedan causar. El presente artículo describe la metodología diseñada para la realización de diagnóstico de vulnerabilidad del departamento del Chocó y a partir de la cual se generaron los insumos para construir el plan de adaptación al cambio climático de este departamento. Con lo anterior se genera un aporte que puede ser empleado para cualquier región del país teniendo en cuenta sus particularidades ambientales y sociales.

\section{Metodología}

Estimación de las amenazas por variabilidad climática. La frecuencia en la ocurrencia de eventos climáticos extremos (FOECE) hace referencia al número de veces que ocurre un mismo fenómeno en un espacio geográfico determinado y en un tiempo específico y exige por lo tanto, el cálculo de valores que se encuentran en dependencia del tipo de fenómeno climático, lo que implica la determinación de un valor de esta variable para cada uno de los eventos climáticos cuya vulnerabilidad se desee determinar, lo cual servirá como factor para la determinación de amenazas.

En este sentido, por el momento, dada la mayor importancia de los eventos climáticos extremos: inundaciones, vendavales, deslizamientos de tierra, avenidas torrenciales, marejadas y sequías, tanto por la frecuencia de su ocurrencia como por su efecto sobre la población, la infraestructura, los bosques y otras variables, se hicieron los cálculos del FOECE para estos fenómenos, base para la estimación de la vulnerabilidad y el riesgo, por cada una de las líneas estratégicas definidas en la política de cambio climático.

Según el método propuesto, por ejemplo, si el interés se centra en la determinación del factor de vulnerabilidad y el riesgo a inundaciones en el departamento del Chocó y se desea hacer un análisis por las regiones que lo conforman, habría de calcularse un FOECE para el evento climático inundaciones, y para cada una de las cinco regiones (Atrato, Darién, San Juan, Baudó y costa pacífica), para lo cual se toma el total de eventos climáticos extremos acontecidos en un tiempo en una región y las inundaciones se expresan como un porcentaje de este total de eventos.

Para ilustrar mejor este ejemplo, supongamos que en la región del Atrato sucedieron entre 1935 y 2015 la siguiente cantidad de eventos climáticos extremos: 400 inundaciones, 250 vendavales, 150 


\section{Bioetnia Volumen 12, 2015}

Tabla 1. Calificación del grado de amenaza

\begin{tabular}{cl}
\hline FOECE & Grado de amenaza \\
\hline $00-20$ & Muy bajo \\
$20-40$ & Bajo \\
$40-60$ & Medio \\
$60-80$ & Alto \\
$80-100$ & Muy alto \\
\hline
\end{tabular}

deslizamientos de tierra, 30 avenidas torrenciales, 2 sequías, 18 lluvias intensas. De esta manera de un total de 850 eventos climáticos extremos, $400(44.4 \%)$ corresponde a inundaciones, lo que equivale alFOECE de inundaciones para la región del Atrato. Obtenidos los valores de FOECE, la amenaza por evento climático se expresa en función de ese dato como se muestra en la Tabla 1.

Análisis de vulnerabilidad a eventos climáticos extremos y cambio climático. Para este análisis se propone una estimación de la vulnerabilidad de cada región del departamento a cada uno de los eventos climáticos extremos a partir de la frecuencia con que ocurren desde 1935 hasta la actualidad, y las necesidades básicas insatisfechas, para utilizar esta estimación como punto de partida, en los análisis de vulnerabilidad por línea estratégica de la política, incorporándole las afectaciones sufridas por la población, las viviendas, los cultivos y los bosques, entre otros elementos. La metodología propuesta identifica como unidad de análisis: la frecuencia en la ocurrencia de los eventos hidroclimáticos e hidrometeorológicos extremos y su relación con el índice de necesidades básicas insatisfechas (NBI).

El punto de partida para el análisis de vulnerabilidad es un indicador que se denominará factor de vulnerabilidad, el cual combina dos factores con el mismo valor de ponderación o pesos específico, por una parte, la frecuencia con la que han ocurrido los eventos climáticos extremos asociados con variabilidad climática en el departamento del Chocó, lo cual es un reflejo de la realidad que vive el ente territorial en materia de eventos que influyen directa o indirectamente en sus pobladores y poblaciones, y por otra parte, el índice de necesidades básicas insatisfechas (NBI), en el entendido que la falta de condiciones básicas de la población la hacen más vulnerable a los efectos de los eventos climáticos extremos y afectan su capacidad de respuesta frente al fenómeno. En consecuencia, el factor de vulnerabilidad será una función de la frecuencia de los eventos climáticos extremos y las necesidades básicas insatisfechas, la cual se expresa en los siguientes términos:

$$
F V=(F O E C E+\mid N B I) \div 2
$$

Donde todas las variables se expresan en porcentaje y representan:

$\mathrm{FV}=$ Factor de vulnerabilidad

FOECE $=$ Frecuencia en la ocurrencia de eventos climáticos extremos

INBI=Índice de necesidades básicas insatisfechas

El índice de necesidades básicas insatisfechas (INBI) de la región del Atrato, se expresa como un promedio de las NBI del total (11) de los municipios del departamento del Chocó. Si el promedio de las NBI para los 11 municipios que conforman la región Atrato fuese de $77.5 \%$, este dato correspondería al INBI de la región. Como resultado de la aplicación de la ecuación expuesta para el cálculo del factor de vulnerabilidad, a manera de ejemplo, se tendría:

$$
F V=(44.4 \%+77.5 \%) 2=60.9 \%
$$

Como se dijo antes, el factor de vulnerabilidad no representa la vulnerabilidad, solo sirve de base para calcularla, pues la vulnerabilidad se expresa en función de lo que ocurra en el departamento del Chocó por cada una de las líneas estratégicas de la política. Sin embargo, de desearse hacer una interpretación de la vulnerabilidad genérica del Chocó por regiones o por municipios se podría acudir al factor de vulnerabilidad, con la aplica- 
Tabla 2. Clasificación general de la vulnerabilidad o grado de vulnerabilidad general

\begin{tabular}{cl} 
Vulnerabilidad (vu\%) & Grado de vulnerabilidad \\
\hline $00-20$ & Muy baja \\
$20-40$ & Baja \\
$40-60$ & Media \\
$60-80$ & Alta \\
$80-100$ & Muy alta \\
\hline
\end{tabular}

ción de la Tabla 2.

La vulnerabilidad a un determinado evento climático extremo, se expresará como el promedio del factor de vulnerabilidad a ese evento climático y los efectos sobre la variable correspondiente a la línea estratégica de la política que se pretende analizar(FELE). La lógica de cálculo del FELE es la misma que se siguió para la determinación del FOECE, es decir, el FELE para el análisis de la vulnerabilidad a inundaciones desde la perspectiva de biodiversidad y servicios ecosistémicos, se estimará como un porcentaje de la superficie de bosques afectados por inundaciones en la región o municipio, sobre el total de afectados por eventos climáticos. En las Tablas 3 a 6 se presentan las variables a considerar y los valores del factor de efecto por línea estratégica, por regiones del departamento del Chocó.

Entonces, la vulnerabilidad se calcula haciendo uso de la siguiente expresión matemática, que tiene como variables el factor de vulnerabilidad (FV) y el factor de efecto por línea estratégica (FELE).

$$
\mathrm{V} \%=(\mathrm{FV} \%+\mathrm{FELE} \%) \div 2
$$

\section{Análisis de la vulnerabilidad por componente} estratégico. La vulnerabilidad del departamento del Chocó ante los efectos extremos de la variabilidad y el cambio climático, son explicados principalmente por el alto grado de susceptibilidad (ubicación geográfica de las comunidades) lo que hace que la manifestación intempestiva de los fenómenos hidroclimáticos e hidrometeorológicos extremos impacten fuertemente sobre las poblaciones y sus bienes materiales, además de la baja resiliencia (o capacidad de absorber una perturbación sin ver afectado el desarrollo de sus estructuras y funcionalidades).

Lo anterior es precisamente porque el mayor porcentaje de la población chocoana basa su economía en actividades de subsistencia, dedicadas al

Tabla 3. Variables a considerar para el cálculo de la vulnerabilidad por línea estratégica

\begin{tabular}{ll|}
\hline \multicolumn{1}{c}{ Línea estratégica de la política } & \multicolumn{1}{c}{$\begin{array}{c}\text { Variable a considerar para el análisis de la } \\
\text { vulnerabilidad }\end{array}$} \\
\hline Biodiversidad y servicios ecosistémicos & $\begin{array}{l}\text { Superficie en hectáreas de bosques afectadas por el } \\
\text { respectivo evento climático extremo }\end{array}$ \\
\hline Gestión del recurso hídrico & $\begin{array}{l}\text { Efecto sobre la disponibilidad en cantidad y calidad de } \\
\text { agua para el consumo humano }\end{array}$ \\
\hline Gestión integral de zonas marino-costeras, insulares y & $\begin{array}{l}\text { Erosión y calidad del agua marino-costera, insulares y } \\
\text { oceánica }\end{array}$ \\
\hline sceánicas & $\begin{array}{l}\text { Nivel general de nutrición de los pobladores y disponibili- } \\
\text { dad de alimentos }\end{array}$ \\
\hline Sectores productivos e infraestructura básica & $\begin{array}{l}\text { Viviendas destruidas y afectadas por la ocurrencia del } \\
\text { evento climático extremo }\end{array}$ \\
\hline Sistemas de ciudades & Número de personas afectadas por la ocurrencia de un \\
& evento climático extremo \\
\hline
\end{tabular}


Bioetnia Volumen 12, 2015

Tabla 4. Valores de FELE por evento climático extremo y por regiones del Chocó para biodiversidad y servicios ecosistémicos

\begin{tabular}{lrrrrc}
\hline \multirow{2}{*}{ Evento climático } & \multicolumn{5}{c}{ Región } \\
\cline { 2 - 6 } & Atrato & Baudó & Darién & Pacífico & San Juan \\
\hline Inundación & 58,4 & 87,3 & 100,0 & 50,8 & 85,9 \\
Vendaval & 33,1 & 12,7 & 0,0 & 49,2 & 14,1 \\
Deslizamiento & 0,0 & 0,0 & 0,0 & 0,0 & 0,0 \\
Avenidas torrenciales & 8,5 & 0,0 & 0,0 & 0,0 & 0,0 \\
Marejadas & 0,0 & 0,0 & 0,0 & 0,0 & 0,0 \\
Sequías & 0,0 & 0,0 & 0,0 & 0,0 & 0,0 \\
\hline
\end{tabular}

Tabla 5. Valores de FELE por evento climático extremo y por regiones del Chocó para sectores productivos e infraestructura básica

\begin{tabular}{lrrrrc}
\hline \multirow{2}{*}{ Evento climático } & \multicolumn{5}{c}{ Región } \\
\cline { 2 - 6 } & Atrato & Baudó & Darién & Pacífico & San Juan \\
\hline Inundación & 72,7 & 85,3 & 83,4 & 78,7 & 82,5 \\
Vendaval & 25,2 & 14,6 & 16,6 & 19,5 & 12,3 \\
Deslizamiento & 0,7 & 0,0 & 0,0 & 0,2 & 0,4 \\
Avenidas torrenciales & 1,4 & 0,1 & 0,0 & 0,3 & 0,4 \\
Marejadas & 0,0 & 0,0 & 0,0 & 1,3 & 0,0 \\
Sequías & 0,0 & 0,0 & 0,0 & 0,0 & 0,0 \\
\hline
\end{tabular}

Tabla 6. Valores de FELE por evento climático extremo y por regiones del Chocó para sistemas de sociedades

\begin{tabular}{lrrrrr}
\hline \multirow{2}{*}{ Evento climático } & \multicolumn{5}{c}{ Región } \\
\cline { 2 - 6 } & Atrato & Baudó & Darién & Pacífico & San Juan \\
\hline Inundación & 74,9 & 91,5 & 88,3 & 87,1 & 83,1 \\
Vendaval & 11,1 & 8,4 & 10,5 & 10,0 & 5,2 \\
Deslizamiento & 0,8 & 0,0 & 0,0 & 0,6 & 3,6 \\
Avenidas torrenciales & 0,3 & 0,0 & 1,2 & 0,1 & 0,0 \\
Marejadas & 0,0 & 0,0 & 0,0 & 0,9 & 0,0 \\
Sequías & 12,8 & 0,0 & 0,0 & 0,1 & 6,7 \\
\hline
\end{tabular}

desarrollo de actividades del sector agropecuario, la pesca, la caza, el aprovechamiento forestal, la minería, actividades pecuarias entre otras; donde las condiciones del clima influyen determinantemente el desarrollo satisfactorio de estas labores; por consiguiente, las fases extremas de la variabilidad climática impactan de manera negativa a las comunidades y sus labores socioeconómicas en el departamento.

Un análisis exhaustivo de la vulnerabilidad implica la combinación del factor de vulnerabilidad a un determinado evento climático extremo y la consideración de sus efectos sobre las variables relacionadas con la línea estratégica de la política 
de cambio climático de interés. El análisis integral de la vulnerabilidad se hará a partir de los resultados obtenidos con la aplicación de las tablas y figuras anteriores, superponiéndole información relativa a los escenarios climáticos tendenciales y los aspectos biológicos, físicos y socioculturales del espacio geográfico objeto de análisis.

Evaluación del riesgo climático del Chocó. El riesgo de la población expuesta ante amenazas de la variabilidad climática en los últimos 80 años en el departamento del Chocó, se debe sobre todo a la ubicación en áreas de riesgo de las comunidades afrodescendientes, colonos e indígenas. Entre los años 1935 y 2015 se han presentado en el departamento del Chocó 1.074 eventos extremos de origen hidroclimáticos e hidrometeorológicos, dentro de los que se encuentran: avalanchas, avenidas torrenciales, deslizamientos, incendios forestales, inundaciones, lluvias intensas, mar de leva, marejadas, sequías, tempestades, tormentas eléctricas y vendavales. Los eventos más frecuentes son las inundaciones (646), los vendavales (218) y los deslizamientos de tierras (157), casos reportados, que abarcan más del $95 \%$ de los eventos hidrometeorológicos e hidroclimáticos extremos acaecidos en el departamento del Chocó, lo que a nivel cualitativo es un indicador de que existe riesgo por eventos climáticos marcados.

El riesgo climático se define, como la magnitud probable esperada de daño o fallas de uno o más elementos de un sistema, ocasionados por el desencadenamiento de fenómenos peligroso sobre la población y los bienes particularmente. La fórmula para calcular el riesgo por variabilidad climática desde el punto de vista cuantitativo es:

$$
\mathrm{RVC}=(\mathrm{FOECE} \times \mathrm{V}) / 100
$$

Dónde:

RVC=Riesgo por variabilidad climática

FOECE $=$ Frecuencia en la ocurrencia de eventos climáticos extremos

$\mathrm{V}=$ Vulnerabilidad (resulta de promediar el factor de vulnerabilidad y el factor FELE)

Capacidad de adaptación del departamento del Chocó al cambio climático. La estimación de la capacidad de adaptación del departamento del Chocó al cambio climático se plantea para seis componentes estratégicos:

Biodiversidad y servicios ecosistémicos

- Gestión integral de zonas marino-costeras, insulares y oceánicas

- Gestión del recurso hídrico

- Sectores productivos e infraestructura básica

- Sistemas alimentarios

- Sistema de ciudades

Los puntajes obtenidos en la calificación de la capacidad de adaptación al cambio climático para cada línea estratégica o componente variará entre 0 puntos y 100 puntos, los cuales serán utilizados para su caracterización (Tabla 7).

Tabla 7. Caracterización de la capacidad de adaptación al cambio climático

\begin{tabular}{lr}
\hline Capacidad de adaptación & Puntaje \\
\hline Muy baja & $00-20$ \\
Baja & $20-40$ \\
Media & $40-60$ \\
Alta & $60-80$ \\
Muy alta & $80-100$ \\
\hline
\end{tabular}

La propuesta metodológica se fundamenta en dos consideraciones: la primera que constituya un procedimiento sencillo, fácil de entender y aplicar, y la segunda, que su aplicación requiera de información disponible, confiable y actualizable, de tal manera que se vayan haciendo ajustes que afinen cada vez más el método.

Dentro de cada uno de los componentes o líneas estratégicas se proponen variables que serán definidas en la medida en que se vaya desarrollando el proceso metodológico propuesto.

Biodiversidad y servicios ecosistémicos

- Existencia de figuras de conservación de ecosistemas 


\section{Bioetnia Volumen 12, 2015}

- Magnitud de la cobertura de bosques

- Tipos de uso de la tierra

La definición de estas variables tiene como fundamento la consideración que la biodiversidad y los servicios ecosistémicos de una región tendrán capacidad de adaptación al cambio climático, en la medida en que cuenten, en relación con su tamaño, con importantes coberturas de bosques naturales que sustenten la existencia de la biodiversidad regional, que estos bosques cuenten con mecanismos eficaces para su conservación, y que no existan presiones sobre el territorio por el desarrollo de actividades productivas altamente contaminantes o impactantes del medio natural.

Para la calificación de la capacidad de adaptación de la biodiversidad y los servicios ecosistémicos de las regiones del departamento del Chocó, a cada una de las variables, magnitud de la cobertura de bosques, existencia de figuras de conservación y tipos de uso de la tierra, se les asignará un puntaje que variará entre un mínimo de 0.00 puntos y un máximo de 33.33 puntos, de la siguiente manera:

Cobertura de bosques. Esta variable corresponde a la superficie de coberturas boscosas que presenta una región expresada como un porcentaje de la superficie total de esa región; los puntajes que se asignan para su medición tendrán como referencia la región de Pacífico que presenta la mayor cobertura de bosques en términos porcentuales y a la cual se le asignará el máximo puntaje de 33,33; las otras regiones se calificarán proporcionalmente.

Superficie de áreas protegidas. Esta variable hace referencia a la existencia dentro de una región, de áreas de conservación debidamente registradas en el RUNAP (Registro Único Nacional de Áreas Protegidas). Se valorará tomando como punto de referencia $29,6 \%$ de la respectiva superficie prioritaria de conservar, según el estudio de zonificación ambiental de la Reserva Forestal del Pacífico adelantado por el IIAP y el MADS, resultado de tomar la región del Darién como base, por ser esta la que tiene la mayor superficie de áreas protegidas en relación con la superficie total con prioridad de conservación. Los puntajes se otorgarán así: Si la superficie en áreas protegidas de una región inscritas en el RUNAP es igual a $29,6 \%$ de la superficie de esa región con prioridad de conservación, se le asignan 33,33 puntos como máximo; si está por debajo se le asignará puntaje proporcionalmente.

Tipos de uso de la tierra. Esta variable hace referencia a la manera como en la actualidad son utilizadas las tierras de una región, poniendo énfasis en el desarrollo de actividades productivas de alto impacto para la existencia de los bosques y en consecuencia para la conservación de la biodiversidad; se tendrán en cuenta las cuatro actividades productivas mayormente aportantes a la reducción de la superficie boscosa en el departamento del Chocó, minería, agropecuaria, cultivos de uso ilícito y silvícola.

Se partirá de un máximo de 33,33 puntos para aquellas regiones que no presenten ninguna de estas actividades de alto impacto sobre la estabilidad de los bosques, hasta un mínimo de 0 puntos para las regiones que tengan todas estas actividades. Por la presencia en la región de una o varias de las actividades productivas relacionadas se restarán puntajes, así: por minería 15 puntos, por actividades agropecuarias de palma africana y ganadería 10 puntos, por cultivos de uso ilícito 5 puntos y por aprovechamiento forestal 3,33 puntos.

Gestión del recurso hídrico

- Disponibilidad de agua

- Calidad del agua disponible

- Existencia de planes de manejo de cuencas hidrográficas

La definición de estas variables tiene su fundamento en que la capacidad de adaptación del recurso hídrico de una región específica del departamento del Chocó a los efectos de los eventos climáticos extremos y el cambio climático será más efectiva, en la medida en que existan fuentes hídricas que posibiliten una oferta permanente 
de agua siempre mayor a la demanda, que el agua disponible sea de calidad para las muy diversas actividades regionales que dependen de este recurso y constituyen toda una cultura departamental alrededor de él, y que las cuencas hidrográficas en las cuales se deposita la oferta hídrica cuenten con planes de manejo formulados y en implementación.

Para la calificación de la capacidad de adaptación de los recursos hídricos de las regiones del departamento del Chocó, a cada una de las variables, disponibilidad de agua, calidad del agua disponible y existencia de planes de manejo de cuencas hidrográficas en implementación, se les asignará un puntaje que variará entre un mínimo de 0,00 puntos y un máximo de 33,33 puntos para posibilitar un tope de 100 puntos, de la siguiente manera:

Disponibilidad de agua. La variable disponibilidad de agua se entiende como la diferencia entre la oferta y la demanda de agua en una región, se asignará puntajes tomando como base la región Atrato, la cual presenta la mayor relación oferta/ demanda $(6,25)$ y en consecuencia se le otorgará el puntaje máximo de 33,33 puntos. Los otros puntajes se otorgarán de manera proporcional.

Calidad del agua disponible. Esta variable hace referencia a la calidad que tenga el agua disponible en una región, no solo para el consumo humano, sino también para todos los usos que esta represente. Se partirá de otorgar el mayor puntaje $(33,33)$ a las regiones en cuyas cuencas hidrográficas de sus principales fuentes hídricas no se desarrollen actividades de alto impacto que deterioren de manera significativa la calidad física, química y biológica de los cuerpos de agua, en su orden para el caso del Chocó, la minería mecanizada, la disposición directa de excretas y la disposición directa de residuos sólidos.

En la medida en que estas actividades se realicen sobre los afluentes o fuentes hídricas principales de la región se restará al puntaje máximo de 33,33 los siguientes puntajes: por minería 13,33 puntos, por disposición final de excretas sobre la fuente 10,00 puntos, $\mathrm{y}$ por disposición final directa de residuos sólidos sobre la fuente 10,00 puntos, hasta llegar a 0,00 puntos si se presentan simultáneamente las tres actividades.

Existencia de planes de manejo de cuencas hidrográficas. Esta variable se refiere a que en las cuencas hidrográficas principales de cada región o sus afluentes principales, existan planes de manejo de cuencas formulados y en implementación, con lo que se piensa se podría ejercer control sobre los motores que producen deterioro a la calidad del recurso hídrico. Si las cuencas hidrográficas principales o sus afluentes más importantes tienen planes de manejo formulados y en implementación se les otorgará 33,33 puntos; si solo se encuentran formulados se asignarán 16,66 puntos, si no existen planes de manejo formulados se asignará 0,00 puntos.

Gestión integral de zonas marinas y costeras

- Existencia de áreas marinocosteras protegidas

- Calidad de las aguas marinocosteras

- Planes de manejo de Unidades Ambientales Costeras

Este componente es exclusivo de las regiones Darién y costa pacífica únicas del departamento del Chocó en las cuales existen zonas marinas y costeras. La definición de estas variables tiene como fundamento la necesidad de garantizar calidad de las aguas marinas para la conservación de la biodiversidad de los mares y la prestación de sus servicios ecosistémicos, lo cual tiene impacto sobre muchas actividades que en términos culturales y productivos dependen de los mares y las costas, para lo cual será necesario que los ecosistemas marino costeros estén protegidos mediante alguna figura de conservación prevista por la legislación colombiana y que las Unidades Ambientales Costeras estén debidamente delimitadas, caracterizadas y con planes de manejo formulados y en implementación.

Para la calificación de la capacidad de adap- 


\section{Bioetnia Volumen 12, 2015}

tación al cambio climático de los mares y costas de las regiones del departamento del Chocó, a cada una de las variables, existencia de áreas marino-costeras protegidas, calidad de las aguas marino-costeras y existencia de planes de manejo de unidades ambientales costeras formulados y en implementación, se les asignará un puntaje que variará entre un mínimo de 0,00 puntos y un máximo de 33,33 puntos para posibilitar un tope de 100 puntos, de la siguiente manera:

Existencia de áreas marino-costeras protegidas. Esta variable hace referencia a la existencia en una región con mares y costas, de zonas marino-costeras protegidas, debidamente declarada por la autoridad ambiental del departamento y relacionada en el Registro Único Nacional de Áreas Protegidas. Si una región posee dos o más áreas marino-costeras protegidas se le asignará un puntaje de 33,33 puntos; si solo posee una, se le otorgarán 16,66 puntos; si no posee ninguna 0,00 puntos.

Calidad de las aguas marino costeras. Hace referencia a la disponibilidad de agua de calidad para las actividades desarrolladas alrededor de los ecosistemas marinos y costeros; se propone estimar su calificación de manera similar a la utilizada para la calidad del agua continental, así: disposición final directa de excretas al mar, disposición final de residuos sólidos al mar y aporte de sedimentos por parte de fuentes hídricas superficiales que vierten sus aguas al mar, producto de actividades productivas.

Se partirá de un máximo de 33,33 puntos y se restará puntajes a las regiones, de la siguiente manera: por aportes de sedimentos por minería proveniente de afluentes que drenan al mar, 13,33 puntos; por disposición final de excretas sobre la fuente 10,00 puntos; y por disposición final directa de residuos sólidos sobre la fuente 10,00 puntos, hasta llegar a 0,00 puntos si se presentan simultáneamente las tres actividades.

Planes de manejo de unidades ambientales costeras. Se refiere a la existencia de planes de manejo de las unidades ambientales costeras debidamente formulados y en implementación, condición para la cual se asignará a la región un máximo de 33,33 puntos; si los planes de manejo solo se encuentran formulados se asignarán 16,66 puntos; si no existen planes de manejo formulados la calificación será de 0,00 puntos.

Sectores productivos e infraestructura básica

- Disponibilidad de recursos naturales

- Sistemas de extracción de los recursos naturales

- Existencia y estado de aeropuertos o vías que comunican la región con el interior

- Existencia y estado de sistemas de transporte intermunicipales en la región

Con la definición de las variables conformantes de este componente, se estima que la adaptación al cambio climático de los sectores productivos y la infraestructura básica de una región se dará en la medida que se cuente con una base de recursos naturales que provean las materias primas suficientes para mantener activo cada sector productivo, el sistema que se utilice para la extracción de los recursos naturales sea de bajo impacto para el medio natural y el recurso mismo, y los aeropuertos o vías que comunican a la región con otras regiones del departamento o con el interior del país se encuentren en condiciones óptimas, lo mismo que las vías que internamente comunican a los diversos municipios de una región determinada.

Cuando cada una de estas variables presente las mejores condiciones para mantener los procesos de adaptación recibirá 25 puntos, hasta completar un máximo de 100; cuando ocurra el caso contrario recibirán un mínimo de 0,00 puntos; la calificación se hará de la siguiente manera:

Disponibilidad de recursos naturales. Se tomarán en cuenta los sectores productivos más importantes del departamento del Chocó, pesca marina y continental, silvicultura, minería y agropecuario, por lo que la abundancia de recursos naturales por cada sector productivo recibirá un puntaje de 6,25 puntos, hasta completar 25 para 


\section{Herramienta metodológica y estimación de eventos climáticos. W Klinger Brahan, L Vargas Porras}

aquellas regiones con mayor abundancia de los mismos en todos los sectores productivos. La abundancia del recurso natural en cada sector productivo se calificará como nula (0), baja (2), media (4) o alta $(6,25)$.

Sistemas de extracción. Se presume una mayor capacidad de adaptación al cambio climático cuando los sistemas de extracción utilizados para proveerse de materias primas sean de bajo impacto para el medio natural, lo cual contribuiría a la permanencia y estabilidad del recurso natural. En la calificación de esta variable se llegará a un máximo de 25 puntos, los cuales se obtendrán si en los cuatro sectores productivos (pesca, silvicultura, agropecuario y minería) se utilizan sistemas de extracción de impacto nulo sobre el recurso y el medio natural. Los puntajes asignables por cada sector productivo, son de $6,25,4,0,2,0$ y 0,0 puntos si los sistemas de extracción del recurso natural son de impacto nulo $(6,25)$, bajo impacto $(4,0)$, impacto medio $(2,0)$ y alto impacto $(0,0)$.

Estado de los aeropuertos o vías que comunican la región con otras regiones o el interior del país. Para esta variable se parte del supuesto que el estado de la infraestructura posibilita el sostenimiento de los sectores productivos en tanto facilita la comercialización de productos y el intercambio comercial con otras regiones del departamento y con el interior del país u otros lugares donde se puedan mercadear los productos. Se proponen cuatro categorías: inexistencia de aeropuertos en la región 0,0 puntos, aeropuertos en mal estado 5,0, aeropuertos en regular estado 15 y aeropuertos en buen estado 25 puntos.

Existencia y estado de las carreteras que comunican internamente la región. Se parte del supuesto que habrá mayores posibilidades de intercambio comercial y crecimiento económico, si internamente en las regiones se garantiza la infraestructura necesaria para la comercialización de productos entre los diferentes municipios que conforman las regiones. Se proponen cuatro categorías: inexistencia de sistema de transporte intermunicipal en la región 0,0 puntos, mal estado de los sistemas de transporte intermunicipal en la región 5, regular estado de los sistemas de transporte intermunicipal 12,5, y buen estado del sistema de transporte intermunicipal de la región 25 puntos.

\section{Sistemas alimentarios}

- Disponibilidad de tierras para el cultivo de especies vegetales alimenticias

- Disponibilidad de cuerpos de agua con buena calidad biológica

- Presencia regional de especies invasoras o inhibidoras de la fauna y flora propias

- Tráfico ilegal de fauna silvestre utilizada para alimentación

La introducción de estas cuatro variables para la medición de la capacidad de adaptación al cambio climático de los sistemas alimentarios del departamento del Chocó, supone que esta capacidad está conectada con la posibilidad de producción de alimentos, lo cual no sería posible sin tierras disponibles para tal propósito; que ante la cultura de alto consumo de recursos pesqueros como fuente de alimentación, también se debe disponer de cuerpos de agua con suficiente calidad para garantizar un hábitat adecuado de especies propias de la región; que debe restringirse el uso de especies invasoras o inhibidoras cuyo comportamiento incida en altos niveles de reducción de los recursos genéticos propios del departamento tanto en la florístico como en lo fáunico; y que no debe existir tráfico ilegal de fauna silvestre, en tanto esta tiene una gran importancia alimenticia para los habitantes de la región.

Cada una de las variables, disponibilidad de tierras para el cultivo de especies vegetales alimenticias, disponibilidad de cuerpos de agua con suficiente calidad biológica para la vida de especies del alto consumo humano, uso de especies adecuadas o no invasores ni inhibidoras de la fauna y la flora propias del Chocó, y el tráfico ilegal de fauna silvestre utilizada para alimentación, tendrá un máximo de 25 puntos hasta 


\section{Bioetnia Volumen 12, 2015}

completar un total de 100 para una capacidad de adaptación perfecta, así:

Disponibilidad de tierras para el cultivo de especies vegetales alimenticias. Esta variable se expresará como la superficie territorial de una región apta para cultivos en hectáreas, expresada como un porcentaje de la superficie total de la región. Si en una región determinada, la superficie apta para cultivos es $\geq 25 \%$ de la totalidad de las tierras de la región, se asignará a la variable 25 puntos como valor máximo, mientras que si la superficie de tierras aptas para cultivo es $0 \%$, se le asignará un 0 . Las superficies que oscilen entre $0 \%$ y $25 \%$ recibirán puntajes proporcionales; 1 punto por cada $1 \%$ de superficie apta para cultivos.

Disponibilidad de cuerpos de agua con suficiente calidad biológica para la vida de especies de alto consumo humano. Hace referencia a cuerpos de agua tanto marinos como continentales, en el entendido que los peces en el departamento del Chocó son tal vez la mayor fuente de proteína animal. Si en una región existen mares más ciénagas con buena calidad biológica de sus aguas se le asignarán 25 puntos; si con esta misma condición o características existen solo mares 20 puntos, si existen solo ciénagas 15 puntos, si existen solo ríos 10 puntos, si existen otros sistemas de producción de peces 5 puntos.

Presencia de especies invasoras o inhibidoras de la fauna y la flora propia del departamento. Esta presencia puede disminuir drásticamente las poblaciones de fauna y flora nativa que revista importancia para el consumo humano y aporte a la nutrición. Si una región tiene presencia de especies tanto de fauna como de flora que restrinjan el desarrollo normal de las poblaciones nativas se le asignará 0,0 puntos, si tiene presencia de especies que reduzcan o restrinjan el desarrollo de poblaciones de especies de fauna solamente o de flora solamente se le asignarán 12,5, si no hay presencia de especies invasoras o inhibidoras del desarrollo normal de poblaciones nativas se le asignarán 25 puntos.
Tráfico ilegal de fauna silvestre utilizada para alimentación. El tráfico ilegal de fauna silvestre contribuye de manera significativa al agotamiento de especies y reducción de poblaciones biológicas, que han proveído por mucho tiempo recursos para la alimentación de los pobladores del departamento del Chocó. Si el tráfico ilegal de especies de fauna en una región no existe se le asignará 25 puntos, si existe tráfico ilegal de hasta dos especies se le asignarán 12,5 , si existe tráfico ilegal de más de dos especies se le asignará 0,0 puntos.

Sistema de ciudades

- Disponibilidad y acceso a servicios públicos básicos

- Infraestructura social

- Morbilidad asociadas con variabilidad climática

- Desplazamiento forzado de personas asociado con violencia

La definición de las variables que conforman el sistema de ciudades supone que la adaptación más efectiva al cambio climático ocurrirá si la población dispone y accede a servicios públicos de calidad tanto en la prestación como en el servicio mismo, si cuenta con infraestructura social representada en escuelas, colegios, universidades, centros de salud, clínicas y hospitales, si la morbilidad asociada con fenómenos de variabilidad climática es bajo y no existe desplazamiento forzado de personas atribuibles a fenómenos de violencia.

Cada una de estas variables recibirá un puntaje máximo de 25 y un mínimo de 0,0 para la calificación de la capacidad de adaptación del sistema de ciudades para cualquiera de las regiones que conforman el departamento del Chocó; la asignación de puntajes se hará atendiendo los siguientes criterios:

Disponibilidad y acceso a servicios públicos básicos. Se consideran los servicios públicos de agua, energía eléctrica, alcantarillado, disposición de residuos sólidos, telefonía y conectividad 
electrónica. Por la disponibilidad y acceso de la población de manera permanente a servicios públicos se otorgarán puntajes hasta completar 25, así:

- Agua potable: 5 puntos

- Energía eléctrica: 5 puntos

- Alcantarillado: 5 puntos

- Disposición de residuos sólidos: 5 puntos

- Telefonía: 3 puntos

- Conectividad electrónica: 2 puntos

Disponibilidad y acceso a la infraestructura social. Si en una región hay presencia, disponibilidad y acceso al sistema educativo en todos sus niveles, se le otorgarán hasta 15 puntos; si hay presencia, disponibilidad y acceso a centros de salud y hospitales, se le asignarán hasta 10 puntos, así:

- Básica primaria: 5 puntos

- Básica secundaria: 5 puntos

- Superior: 5 puntos

- Centros de salud: 5 puntos

- Hospitales: 5 puntos

Morbilidad asociada con variabilidad climática. Se estimará por el incremento en los casos de enfermedades asociadas con variabilidad climática teniendo como período de análisis los últimos 10 años, específicamente las siguientes: paludismo, dengue, afecciones de la piel, enfermedades respiratorias agudas y enfermedades gastrointestinales. Se partirá de un total de 25 puntos y se restarán 5 por el incremento en una región determinada de alguna de estas enfermedades.

Desplazamiento forzado. Este fenómeno nocivo para la construcción social de ciudades o poblados, tendrá un período de análisis de 10 años y se evaluará por el incremento del número de personas desplazada por violencia en cada región, regiones con decrementos o estabilidad en el período de análisis tendrá un puntaje de 25; regiones con incrementos del desplazamiento de personas hasta el $20 \%$ en el período de análisis tendrán un puntaje de 20; regiones con incrementos en el desplazamiento de personas entre
$20 \%$ y $40 \%$ tendrán un puntaje de 15 ; regiones con incrementos del desplazamiento forzado de personas entre $40 \%$ y $60 \%$ tendrán un puntaje de 10; regiones con incrementos del desplazamiento forzado de personas entre $60 \%$ y $80 \%$ tendrán un puntaje de 5 puntos; y regiones con incrementos del desplazamiento forzado de personas por encima de $80 \%$ tendrán un puntaje de 0 .

\section{Resultados}

Aplicando la metodología construida se presentan los resultados obtenidos para la amenaza inundaciones que es una de las más frecuentes en el territorio del departamento del Chocó. En la Tabla 8, se relacionan los datos calculados para el FOECE y el grado de amenaza en cada una de las subregiones.

Tabla 8. Grado de amenaza por inundaciones por regiones del Chocó

\begin{tabular}{lcl}
\hline Región & FOECE \% & Grado de amenaza \\
\hline Atrato & 58,6 & Medio \\
Baudó & 67,5 & Alto \\
Darién & 77,3 & Alto \\
Pacífico & 50,0 & Medio \\
San juan & 58,6 & Medio \\
\hline
\end{tabular}

Con fundamento en este proceso metodológico se calcularon los factores de vulnerabilidad de las cinco regiones del Chocó a este evento climático de mayor importancia en el período 1935-2015. En la Tabla 9, se presentan los valores de factor de vulnerabilidad, que servirán como insumo fundamental en el cálculo de la vulnerabilidad por línea estratégica de la política en cada región.

Utilizando el factor de vulnerabilidad, para hacerse a una idea de la vulnerabilidad general del Chocó frente a los eventos climáticos extremos de mayor importancia ocurridos entre 1935 y 2015, se obtienen los resultados que se muestran en la Tabla 10, los cuales permiten estimar, para cada una de las regiones del departamento, este 


\section{Bioetnia Volumen 12, 2015}

Tabla 9. Factor de vulnerabilidad para inundaciones por regiones del Chocó

\begin{tabular}{llll}
\hline Región & FOECE \% & INBI \% & FV \% \\
\hline Atrato & 58,6 & 76,9 & 67,7 \\
Baudó & 67,5 & 98,6 & 83,0 \\
Darién & 77,3 & 71,4 & 74,4 \\
Pacífico & 50,0 & 61,1 & 55,6 \\
San Juan & 58,6 & 66,8 & 62,6 \\
\hline
\end{tabular}

los bosques de llanuras aluviales ubicados en los ríos más importantes del departamento del Chocó y con recursos boscosos abundantes. Municipios de la cuenca del Atrato y de la región del Darién como Riosucio y Carmen del Darién presentan estas particularidades, al igual que los municipios de la cuenca y región del Baudó, Alto Baudó (Pie Pato) y Medio Baudó (Puerto Meluk).

En la región del Darién, la que presenta la ma-

Tabla 10. Calificación de la vulnerabilidad general a eventos climáticos más frecuentes por regiones del departamento del Chocó a partir del factor de vulnerabilidad

\begin{tabular}{llcllc}
\hline \multirow{2}{*}{ Evento climático } & \multicolumn{5}{c}{ Región } \\
\cline { 2 - 5 } & Atrato & Baudó & Darién & Pacífico & San Juan \\
\hline Inundación & & & & & \\
Factor de vulnerabilidad & 67,75 & 83,05 & 74,39 & 55,56 & 62,65 \\
Vulnerabilidad general & Alta & Muy alta & Alta & Media & Alta \\
\hline
\end{tabular}

importante parámetro.

Con la aplicación de la ecuación propuesta se obtienen los resultados de vulnerabilidad para el evento climático de inundaciones por regiones del Chocó (Tabla 11).

Las inundaciones es el evento climático extremo de mayor importancia en relación con la biodiversidad y los servicios ecosistémicos del Chocó, por la frecuencia de su ocurrencia y su impacto sobre la superficie de bosques del departamento. La calificación de esta vulnerabilidad es de $72,6 \%$, lo que permite categorizarla como de grado ALTO.

Las regiones mayormente afectadas corresponden a Darién $(87,2 \%)$ y Baudó $(85,2 \%)$, se encuentran en grado MUY ALTO de vulnerabilidad de su biodiversidad y servicios ecosistémicos. Las regiones San Juan (74,3\%) y Atrato (63,1\%) presentan un grado de vulnerabilidad ALTO, mientras que la región Pacífico $(53,2 \%)$ resulta la menos vulnerable con un grado de vulnerabilidad MEDIO Estos resultados se explican por los efectos devastadores de las inundaciones sobre yor vulnerabilidad de su biodiversidad y servicios ecosistémicos, se encuentran los municipios de Acandí, Unguía, Riosucio y Carmen del Darién. En términos generales, la región contiene ecosistemas marino costeros en los municipios de Unguía yAcandí, en donde predominan como actividades productivas el turismo y la pesca, así como áreas comunitarias dedicadas a la conservación, a pesar de lo cual algunas prácticas productivas han provocado incendios forestales que profundizan la vulnerabilidad de su biodiversidad y los servicios que esta presta.

También se encuentran en la región del Darién los municipios de Riosucio y Carmen del Darién por excelencia forestales, con bosques de una gran riqueza florística, sometidos al rigor del aprovechamiento forestal, lo que junto con los efectos de las inundaciones sobre los bosques hace más vulnerable su biodiversidad y los servicios que presta. Estos municipios también tienen una amplia dedicación a la pesca continental, porque cuentan con importantes recursos pesqueros que provienen de las extensas ciénagas que se ubican 
Herramienta metodológica y estimación de eventos climáticos. W Klinger Brahan, L Vargas Porras

Tabla 11. Valores de vulnerabilidad por evento climático extremo y por regiones del Chocó

\begin{tabular}{|c|c|c|c|c|c|}
\hline \multirow[t]{2}{*}{ Evento climático } & \multicolumn{5}{|c|}{ Región } \\
\hline & Atrato & Baudó & Darién & Pacífico & San Juan \\
\hline \multicolumn{6}{|l|}{ Inundación } \\
\hline Factor de vulnerabilidad & 67,75 & 83,05 & 74,39 & 55,56 & 62,65 \\
\hline FELE & 58,40 & 87,30 & 100,0 & 50,80 & 85,90 \\
\hline Vulnerabilidad & 63.07 & 85.18 & 87.20 & 53,18 & 74,28 \\
\hline Calificación & Alta & Muy alta & & & \\
\hline \multicolumn{6}{|c|}{$\begin{array}{l}\text { Valores de vulnerabilidad por evento climático extremo y por regiones del Chocó para } \\
\text { sectores productivos e infraestructura básica }\end{array}$} \\
\hline \multicolumn{6}{|l|}{ Inundación } \\
\hline Factor de vulnerabilidad & 67,75 & 83,05 & 74,39 & 55,56 & 62,65 \\
\hline FELE & 72,70 & 85,30 & 83,40 & 78,70 & 82,50 \\
\hline Vulnerabilidad & 70,23 & 84,18 & 78,90 & 67,13 & 72,58 \\
\hline Calificación & Alto & Muy alto & Alto & Alto & Alto \\
\hline \multicolumn{6}{|c|}{$\begin{array}{l}\text { Valores de vulnerabilidad por evento climático extremo y por regiones del Chocó para } \\
\text { sistemas de sociedades }\end{array}$} \\
\hline \multicolumn{6}{|c|}{ Inundación } \\
\hline Factor de vulnerabilidad & 67,75 & 83,05 & 74,39 & 55,56 & 62,65 \\
\hline FELE & 74,90 & 91,50 & 88,30 & 87,10 & 83,10 \\
\hline Vulnerabilidad & 71,33 & 87,28 & 81,35 & 71,33 & 72,88 \\
\hline Calificación & Alta & Muy alta & Muy alta & Alta & Alta \\
\hline \multicolumn{6}{|c|}{$\begin{array}{c}\text { Grado de vulnerabilidad por evento climático extremo y por regiones del Chocó para } \\
\text { biodiversidad y servicios ecosistémicos }\end{array}$} \\
\hline \multicolumn{6}{|l|}{ Inundación } \\
\hline Factor de vulnerabilidad & 67,75 & 83,05 & 74,39 & 55,56 & 62,65 \\
\hline FELE & 58,40 & 87,30 & 100,0 & 50,80 & 85,90 \\
\hline Vulnerabilidad & 63,07 & 85,18 & 87,20 & 53,18 & 74,28 \\
\hline Calificación & Alta & Muy alta & Muy alta & Media & Alta \\
\hline
\end{tabular}

en la cuenca del Atrato y proveen a la región de especies ícticas de gran importancia comercial como el Bocachico, Dentón, Quicharo, Doncella, etc.

La contaminación del río Atrato por efectos de la minería mecanizada y la enorme producción de sedimentos, ha provocado la sedimentación de las bocas del Atrato y la colmatación acelerada de las ciénagas, reduciendo su potencial productivo y poniendo en riesgo la biodiversidad representada en especies dulce acuícolas. Hoy no se discute la notable reducción de importantes especies de peces utilizados para la alimentación como $\mathrm{La}$ Doncella, La Boquiancha y El Veringo, como tampoco la presencia de mercurio en la piel y los tejidos de peces depredadores que indican contaminación inminente de casi toda la cadena trófica y advierte sobre la pérdida de especies que se podría generar en el futuro cercano.

El establecimiento de ganadería y los cultivos de palma africana han promovido la desecación y desviación de fuentes hídricas, el deterioro de la cobertura boscosa y la pérdida acelerada de grandes extensiones de cativo, una comunidad biológica casi homogénea, exclusiva del Chocó, hoy declarada en amenaza por este motivo, además por la intensidad del aprovechamiento al que ha sido sometido, sin que se adelanten procesos de recuperación de una de las especies forestales más importantes del departamento. Adicionalmente, 


\section{Bioetnia Volumen 12, 2015}

estos procesos condujeron a desplazamientos, masacres y muertes con las que se fue buena parte del conocimiento tradicional asociado con el manejo de agrobiodiversidad y los sistemas productivos de la región.

El aprovechamiento forestal selectivo de algunas especies de alto valor comercial ha descremado el bosque, originando, la escases de las especies arbóreas de mayor valor comercial, la reducción de la diversidad de los bosques y la pérdida de relaciones ecológicas de extrema importancia para la conservación de especies, tanto de flora como de fauna. Investigaciones recientes del Instituto de Investigaciones Ambientales del Pacífico han mostrado la reducción significativa de ejemplares de especies forestales en otrora de alta presencia en la región, pino amarillo, guayacán negro, guayacán amarillo, cedro, almendro, entre otros.

El crecimiento acelerado de la superficie dedicada a los cultivos de uso ilícito en la región del Darién, es un claro indicador de la pérdida de bosques primarios y secundarios, pero también del vertimiento de sustancias químicas que pueden generar efectos peligrosos sobre la biodiversidad íctica, además de los efectos sociales y culturales que sufre la población con la ruptura del tejido social, el abandono de la escuela, la pérdida secuencial de actividades productivas autóctonas sostenibles y la vocación productiva regional.

El panorama se torna complicado si al efecto de las inundaciones sobre la biodiversidad y los servicios ecosistémicos de la región del Darién y las prácticas productivas de hoy, las cuales son sin duda motores de pérdida de bosques, se suman los escenarios tendenciales de cambio climático, El incremento en la temperatura y la reducción de la precipitación pronosticada por el IDEAM, podría generar desecación de fuentes hídricas y reducción en la productividad de los bosques, lo que mirado al tenor de la realidad productiva que vive hoy la región, provocaría la pérdida o escases de especies marinas e ictiológicas, así como de importantes extensiones de bosques que reducirían las especies florísticas forestales y no forestales, así como las especies de fauna asociadas con los bosques, tan importantes en la dinámica productiva de las comunidades étnicas y campesinas de la región del Darién.

La región del Baudó está representada por los municipios de Alto Baudó y Medio Baudó, los cuales son por excelencia agrícolas, con predominio de sistemas productivos relativamente limpios y basados en el conocimiento tradicional; el territorio es prácticamente exento de minería mecanizada y esto ha sido así siempre, pero existen evidencia del crecimiento de las extensiones en cultivos de uso ilícito, y aún persisten actividades de aprovechamiento forestal.

Este conjunto de actividades productivas no incidirá tan fuertemente en la vulnerabilidad de la biodiversidad y los servicios ecosistémicos de la región del Baudó, como sí lo hacen con determinación e importancia en la región del Darién; sin embargo, la vulnerabilidad de estas variables a los eventos climáticos extremos, sobre todo a las inundaciones, evidencia la necesidad de tomar medidas que eviten la pérdida acelerada de los bosques y de las ventajas que implica tenerlos. Las tendencias de los escenarios de cambio climático podrían tener los mismos efectos descritos para la región del Darién, manifiestos en reducción de la productividad forestal y la escases de especies de flora y fauna en el Baudó, región azotada por la pobreza extrema de sus pobladores y la presencia de grupos al margen de la ley que ejercen el mayor control del territorio.

La región Atrato está integrada por $11 \mathrm{mu}-$ nicipios, los cuales presentan poblaciones con elementos culturales y actividades productivas variadas, que pueden generar un efecto distinto sobre la vulnerabilidad de la biodiversidad y los servicios ecosistémicos de la región, el cual se clasifica en grado ALTO. Los municipios que integran la región son: Carmen de Atrato, Quibdó, Bagadó, Lloró, Atrato, Medio Atrato, Cértegui, Unión Panamericana, Cantón de San Pablo, Bo- 
jayá y Río Quito.

El Carmen de Atrato tiene en el sector agropecuario su actividad productiva principal, la composición de su población mayoritariamente mestiza no tiene contacto importante con los bosques y su ubicación geográfica en la alta montaña, le permite tener ecosistemas paramunos estratégicos para la producción de agua, lo que hace de la conservación de sus bosques un elemento de extrema importancia en la perspectiva futura. En este momento hay esfuerzos de la autoridad ambiental, la comunidad y la administración municipal por avanzar hacia el establecimiento de áreas de conservación.

Quibdó presenta áreas rurales donde se desarrollan actividades agrícolas, forestales, mineras y cultivos de uso ilícito, situación que sumada a la falta de empleo y oportunidades laborales nos hace mirar con preocupación el futuro inmediato; en este sentido el aporte de Quibdó a la vulnerabilidad de la biodiversidad y los servicios ecosistémicos de la región Atrato, puede resultar importante. En algunas zonas rurales se desarrollan actividades turísticas a pesar de los bajos niveles de desarrollo en materia de infraestructura. La minería ilegal y el poblamiento desordenado de la ciudad de Quibdó han dado al traste con los bosques y la disponibilidad y calidad de los recursos hídricos del municipio.

También exigen una mayor atención las actividades mineras que se adelantan sin responsabilidad ambiental y social en los municipios de Cértegui, Unión Panamericana, Cantón de San Pablo y Río Quito; esta situación es tan dramática e impactante que genera la pérdida más sensible de los bosques en la región, generando efectos no solo sobre la biodiversidad y los servicios ecosistémicos, sino también sobre otros procesos productivos importantes para la economía local y la seguridad alimentaria de las poblaciones étnicas que en gran número habitan en estas municipalidades. La contaminación de los ríos se hace evidente en la presencia de mercurio en varias especies de peces y ha reducido de manera significativa la pesca continental otrora importante en municipios como Río Quito, los mismo ha ocurrido con la pérdida de especies forestales de alto valor comercial que hoy muestran signos de evidente escases.

En municipios como Bojayá y Medio Atrato hay una fuerte actividad productiva ligada con la pesca continental, como también una importante producción agrícola y forestal. Estas actividades se han visto diezmadas por la situación de la minería realizada tanto dentro como fuera del territorio de estos municipios, con impactos sobre los bosques, la calidad del agua y los elementos que le dan identidad cultural a sus pobladores. La lucha por el dominio territorial por parte de grupos armados al margen de la ley ha reducido la presión sobre los bosques desde el aprovechamiento forestal, pero han contribuido a la estabilidad o crecimiento de los cultivos de uso ilícito.

En la Tabla 12 se presenta la calificación de la vulnerabilidad de los seis principales fenómenos extremos en las diferentes regiones del departamento. Se evidencia que el fenómeno de mayor relevancia, tanto por la frecuencia en la ocurrencia como por los impactos que causa sobre la línea específica del sector productivo y la infraestructura básica en el departamento del Chocó, son las inundaciones; en ese sentido, la región del Baudó presenta la mayor vulnerabilidad siendo MUY ALTA en relación con las otras regiones, que en efecto son también de vulnerabilidad ALTA con valores, y para la región de Atrato $(70,2)$, Darién $(78,90$, Pacífico $(67,13)$ y San Juan $(72,58)$. La vulnerabilidad frente a los otros fenómenos como los vendavales, las avenidas torrenciales, las sequías, las marejadas y las lluvias intensas, están categorizadas en niveles BAJOS y MUY BAJOS para todas las regiones.

De acuerdo con lo anterior es preciso resaltar que la región del Baudó, conformada por los municipios del Medio y Alto Baudó centran su actividad productiva en el desarrollo de prácticas 
Bioetnia Volumen 12, 2015

Tabla 12. Vulnerabilidad por regiones del departamento del Chocó

\begin{tabular}{|c|c|c|c|c|c|}
\hline \multirow[t]{2}{*}{ Evento climático } & \multicolumn{5}{|c|}{ Región } \\
\hline & Atrato & Baudó & Darién & Pacífico & San Juan \\
\hline \multicolumn{6}{|l|}{ Inundación } \\
\hline Factor de vulnerabilidad & 67,75 & 83,05 & 74,39 & 55,56 & 62,65 \\
\hline FELE & 72,70 & 85,30 & 83,40 & 78,70 & 82,50 \\
\hline Vulnerabilidad & 70,23 & 84,18 & 78,90 & 67,13 & 72,58 \\
\hline Calificación & Alta & Muy alta & Alta & Alta & Alta \\
\hline \multicolumn{6}{|l|}{ Vendaval } \\
\hline Factor de vulnerabilidad & 47,81 & 63,05 & 44,70 & 46,04 & 40,64 \\
\hline FELE & 25,20 & 14,60 & 16,60 & 19,50 & 12,30 \\
\hline Vulnerabilidad & 36,51 & 38,83 & 30,65 & 32,37 & 26,47 \\
\hline Calificación & Baja & Baja & Baja & Baja & Baja \\
\hline \multicolumn{6}{|l|}{ Deslizamiento } \\
\hline Factor de vulnerabilidad & 48,13 & 51,17 & 36,11 & 34,13 & 44,28 \\
\hline Fele & 0,700 & 0,000 & 0,000 & 0,200 & 0,400 \\
\hline Vulnerabilidad & 24,42 & 25,59 & 18,05 & 17,17 & 22,34 \\
\hline Calificación & Baja & Baja & Muy baja & Muy baja & Muy baja \\
\hline \multicolumn{6}{|l|}{ Avenida torrencial } \\
\hline Factor de vulnerabilidad & 39,19 & 49,92 & 36,89 & 31,45 & 34,88 \\
\hline FELE & 1,40 & 0,100 & 0,000 & 0,30 & 0,400 \\
\hline Vulnerabilidad & 20,30 & 25,01 & 18,45 & 15,88 & 17,64 \\
\hline Calificación & Baja & Baja & Muy baja & Muy baja & Muy baja \\
\hline \multicolumn{6}{|l|}{ Marejada } \\
\hline Factor de vulnerabilidad & & & 35,72 & 34,73 & \\
\hline FELE & & & 0,000 & 1,300 & \\
\hline Vulnerabilidad & & & 17,86 & 18,02 & \\
\hline Calificación & & & Muy baja & Muy baja & \\
\hline \multicolumn{6}{|l|}{ Sequía } \\
\hline Factor de vulnerabilidad & 38,76 & 49,30 & 35,72 & 30,86 & 33,59 \\
\hline FELE & 0,000 & 0,000 & 0,000 & 0,000 & 0,000 \\
\hline Vulnerabilidad & 19,38 & 24,52 & 17,86 & 15,43 & 16,80 \\
\hline Calificación & Muy baja & Baja & Muy baja & Muy baja & Muy baja \\
\hline
\end{tabular}

tradicionales especialmente de cultivos autóctonos utilizados para abastecer las necesidades familiares y locales, de productos como el plátano, maíz, yuca, arroz, cacao y otros árboles frutales los cuales son realizados por lo general sobre la rivera de los ríos, siendo afectado al momento de presentarse una inundación; sumado a este factor se encuentra la ubicación de las viviendas porque para la región del Baudó como para las otras regiones tienen unas afectaciones particulares cuando se presenta la estacionalidad de las precipitaciones las cuales se incrementan con la aparición de fenómenos extremos como las lluvias extremas y las inundaciones prolongadas que desencadenan episodios desastrosos sobre las comunidades que son pequeñas productoras y en 
muchas ocasiones con los único que cuentan es el aprovechamiento de sus tierras y su vivienda, lo que hace que la población sea muy susceptibles a la manifestación de estos fenómenos sobre todo cuando se presentan en los períodos no habituales y son de larga duración.

De los municipios de la región del Baudó, el de mayor impacto por las permanentes inundaciones es el Medio Baudó, sobre todo en la cabecera municipal y sus alrededores; en la región del San Juan, los mayores impactos se presentan en el medio y bajo San Juan al igual que la región del Atrato casi todos los municipios se ven impactados con excepción del Carmen del Atrato y algunas zonas de Quibdó; finalmente la región del Darién con excepción del Acandí todo los municipios que conforman la región se ve afectada por las inundaciones. En todas las regiones el mayor impacto que deja la manifestación de las inundaciones es la limitación de las cadenas productivas como son las labores agrícolas, las pecuarias, la explotación forestal, los pequeños mineros (Tabla 13).

El resultado de la vulnerabilidad para el sistema de ciudades arroja que el evento al cual la población es más vulnerable sin duda alguna son las inundaciones, dejando cantidades de personas afectadas año tras año; lo más grave es que este fenómeno no solo se manifiesta una sola vez al año, sino que es muy frecuente que se produzca en diferentes épocas, causando serios y constantes daños en cultivos, puentes, carreteras, en centros educativos, en puestos de salud y se presenten cierto tipo de enfermedades y demás.

De los resultados de la vulnerabilidad para el sistema de ciudades por regiones, se puede apreciar que la región del Baudó $(87,28)$ y del Darién $(81,35)$ obtuvieron los mayores valores según la metodología propuesta, lo que se traduce en una vulnerabilidad MUYALTA, seguida de la otras regiones las cuales tuvieron una alta vulnerabilidad a las inundaciones, y para las regiones del Atrato $(71,33)$, Pacífico $(71,33)$ y San Juan $(72,88)$.

Para los otros eventos en las cinco regiones como son los vendavales, los deslizamientos, las marejadas y las sequías la vulnerabilidad para el sistema de sociedades, resultaron con valores que oscilan alrededor de $20 \%$ o menores, lo que supone una calificación de Baja a Muy Baja en lo relacionado con la vulnerabilidad para esta línea estratégica específica; esto deduce que aunque existe el riesgo de eventos sobre las dinámicas sociales, siguen siendo más vulnerables a las inundaciones que a fenómenos como los vendavales, las avenidas torrenciales, los deslizamientos, las sequías y las marejada; la fórmula muestra que este comportamiento es similar en las cinco regiones del departamento. Otro aspecto es lo relacionado con el evento de las marejadas, porque a pesar de que es un fenómeno que solo se presentan en las zonas costeras (Pacífico y Darién) presentan Muy Baja vulnerabilidad en lo concerniente a la línea estratégica del sistema ciudades.

Es importante destacar que la metodología empleada para el cálculo de la vulnerabilidad de cada línea estratégica que se plantea en el presente estudio debe disponer de información específica según la temática, la región y los eventos que sean seleccionados, esto con la finalidad de tener el menor grado de incertidumbre con respecto a la vulnerabilidad real.

Para el caso del departamento del Chocó se utilizó la metodología antes descrita; sin embargo, la falta de información específica en cuanto a la gestión del recurso hídrico, marino-costera y los sistemas alimentarios dificultó el análisis de vulnerabilidad para estas temáticas, por lo tanto se deja planteado el proceso y una vez se logre obtener la información solo sea necesario ingresarlo a la fórmula y así poder calcular y analizar la vulnerabilidad de cada línea estratégica para las cinco regiones del departamento del Chocó. 
Bioetnia Volumen 12, 2015

Tabla 13. Valores de vulnerabilidad por evento climático extremo y por regiones del Chocó para los sistemas de ciudades

\begin{tabular}{|c|c|c|c|c|c|}
\hline \multirow[t]{2}{*}{ Evento climático } & \multicolumn{5}{|c|}{ Región } \\
\hline & Atrato & Baudó & Darién & Pacífico & San Juan \\
\hline \multicolumn{6}{|l|}{ Inundación } \\
\hline Factor de vulnerabilidad & 67,75 & 83,05 & 74,39 & 55,56 & 62,65 \\
\hline FELE & 74,90 & 91,50 & 88,30 & 87,10 & 83,10 \\
\hline Vulnerabilidad & 71,33 & 87,28 & 81,35 & 71,33 & 72,88 \\
\hline Calificación & Alta & Muy alta & Muy alta & Alta & Alta \\
\hline \multicolumn{6}{|l|}{ Vendaval } \\
\hline Factor de vulnerabilidad & 47,81 & 63,05 & 44,70 & 46,04 & 40,64 \\
\hline FELE & 11,10 & 8,40 & 10,50 & 10,00 & 5,20 \\
\hline Vulnerabilidad & 29,46 & 35,73 & 27,06 & 28,02 & 22,92 \\
\hline Calificación & Baja & Baja & Baja & Baja & Baja \\
\hline \multicolumn{6}{|l|}{ Deslizamiento } \\
\hline Factor de vulnerabilidad & 48,13 & 51,17 & 36,11 & 34,13 & 44,28 \\
\hline FELE & 0,800 & 0,000 & 0,000 & 0,600 & 3,60 \\
\hline Vulnerabilidad & 24,47 & 25,59 & 18,06 & 17,37 & 23,94 \\
\hline Calificación & Baja & Baja & Muy baja & Muy baja & BAJA \\
\hline \multicolumn{6}{|l|}{ Avenida torrencial } \\
\hline Factor de vulnerabilidad & 39,19 & 49,92 & 36,89 & 31,45 & 34,88 \\
\hline FELE & 0,300 & 0,000 & 1,20 & 0,100 & 0,000 \\
\hline Vulnerabilidad & 19,75 & 24,96 & 19,05 & 15,78 & 17,44 \\
\hline Calificación & Muy baja & Baja & Muy baja & Muy baja & Muy baja \\
\hline \multicolumn{6}{|l|}{ Marejada } \\
\hline Factor de vulnerabilidad & & & 35,72 & 34,73 & \\
\hline FELE & & & 0,00 & 0,90 & \\
\hline Vulnerabilidad & & & 17,86 & 17,82 & \\
\hline Calificación & & & Muy baja & Muy baja & \\
\hline \multicolumn{6}{|l|}{ Sequía } \\
\hline Factor de vulnerabilidad & 38,76 & 49,30 & 35,72 & 30,86 & 33,59 \\
\hline FELE & 12,80 & 0,000 & 0,000 & 0,100 & 6,70 \\
\hline Vulnerabilidad & 25,78 & 24,65 & 17,86 & 15,48 & 20,15 \\
\hline Calificación & Baja & Baja & Muy baja & Muy baja & Baja \\
\hline
\end{tabular}

\section{Riesgo}

En las Tablas 14 y 15 se presentan los cálculos para el grado de riesgo por variabilidad climática, para cada una de las regiones del departamento del Chocó.

La magnitud de riesgo probable esperada de impacto sobre la biodiversidad y servicios ecosistémicos del chocó biogeográfico, por efectos de fenómenos de inundación, tienen un Grado Alto (67.44) para la región del Darién, porque es la zona que presenta mayores áreas inundadas y mayor número de afectados en temporadas de variabilidad climática como los de la "Niña". 
Tabla 14. Comparación del nivel de grado de riesgo por variabilidad climática

\begin{tabular}{cl}
\hline Riesgo (RVC\%) & $\begin{array}{c}\text { Grado de riesgo por } \\
\text { variabilidad climática }\end{array}$ \\
\hline $00-20$ & Muy bajo \\
$20-40$ & Bajo \\
$40-60$ & Medio \\
$60-80$ & Alto \\
$80-100$ & Muy alto \\
\hline
\end{tabular}

Tabla 15. Riesgo para la biodiversidad y servicios ecosistémicos por amenaza de inundación, por región

\begin{tabular}{llcc}
\hline Región & FOECE \% & $\mathbf{V} \%$ & Grado de riesgo \\
\hline Atrato & 58,6 & 63,1 & 36,9 \\
Baudó & 67,5 & 85,2 & 57,5 \\
Darién & 77,3 & 87,2 & 67.4 \\
Pacífico & 50,0 & 53,2 & 26,6 \\
San Juan & 58,5 & 74,3 & 43,5 \\
\hline
\end{tabular}

\section{Estado actual de la capacidad de adaptación al cambio climático en el departamento del Chocó para la línea biodiversidad y servicios ecosistémicos}

De acuerdo con la metodología antes planteada para el análisis de las medidas de adaptación en el departamento delChocó, es necesario describir el estado actual de los sistemas de mayor relevancia en el departamento frente a las posibles proyecciones esperadas en materia de cambio climático; por tal razón, se contempló la caracterización por regiones de la capacidad de adaptación en relación con las líneas estratégicas que se han venido trabajando, la cuales abarcan seis grandes aspectos de gran predominancia sobre el territorio, partiendo de la situación real y actual de todos los municipios que hacen parte de cinco regiones que conforman el territorio chocoano, esto con la finalidad de establecer los mecanismos y acciones más apropiados que garanticen una efectiva adaptación ante los eventos asociados con variabilidad y cambio climático.

La biodiversidad y los sistemas ecosistémicos actualmente se encuentran bajo una fuerte presión por dos razones: en primera instancia por las intensas actividades humanas sobre algunos sectores como la minería, el aprovechamiento forestal y los cultivos de uso ilícitos, los cuales presentan profundas intervenciones sobre el territorio y la superficie boscosa generando un grave retardo en la capacidad de recuperación de algunos sistemas de flora y fauna de gran importancia para el departamento, implicando daños ecosistémicos que en algunas ocasiones son irreparable; a esto se le suma otro parámetro el cual ve limitadas las actividades de las pobladores en las diferentes regiones, como es el caso de la pobreza del departamento, lo que influye en que las mayorías de las actividades tenga que ver con las necesidad de explotar los recursos naturales de manera desordenada, sin una planeación y un control adecuado, para evitar que las afectaciones sean tan marcadas sobre a estos recursos en especial por las grandes afectaciones existentes sobre el bosque natural en los procesos de extracción; por tal razón, las implicaciones son tan desastrosas cuando se evidencian ciertos cambios en las condiciones del clima predominante en un lugar determinado del departamento.

De acuerdo con lo calculado para la capacidad de adaptación sobre el eje de biodiversidad y servicios ecosistemas, actualmente se tiene que la región del Atrato $(45,02)$, San Juan $(50,52)$ y Darién $(57,87)$ tiene una capacidad de adaptación Media, siendo evidente que aunque estas regiones tienen una capacidad de adaptación Media, la región Atrato es la zona más crítica y donde las acciones para esta línea estratégica se debe hacer con mayor énfasis; sin embargo, es válido considerar que aunque también existe una fuerte presión y actividades encaminadas con reducir los impactos y mejorar la capacidad de adaptación 
Bioetnia Volumen 12, 2015

Tabla 16. Capacidad de adaptación: biodiversidad y servicios ecosistémicos

\begin{tabular}{lcccc}
\hline Región & $\begin{array}{c}\text { Cobertura de } \\
\text { bosques }\end{array}$ & $\begin{array}{c}\text { Superficie figuras de } \\
\text { conservación (ha) }\end{array}$ & $\begin{array}{c}\text { Tipos de uso } \\
\text { de la tierra }\end{array}$ & $\begin{array}{c}\text { Capacidad total de } \\
\text { adaptación }\end{array}$ \\
\hline Atrato & 33,14 & 1,88 & 10,00 & 45,02 \\
San juan & 33,22 & 7,30 & 10,00 & 50,52 \\
\hline Baudó & 33,19 & 9,94 & 25,00 & 68,13 \\
Darién & 24,54 & 3,33 & 00,00 & 57,87 \\
\hline Pacífico & 33,33 & 3,27 & 25,00 & 61,60 \\
\hline
\end{tabular}

Fuente: Equipo de trabajo, 2015

en cuanto a la conservación y recuperación de la biodiversidad en materia de cambio climático sobre las otras dos regiones, las necesidades de establecer unas medidas para una eficiente y eficaz adaptación serían menores en comparación con la región del Atrato (Tabla 16).

Las regiones del Pacífico y del Baudó presentan una alta capacidad de adaptación frente a la línea estratégica considerada, siendo esto el resultado de la bajas intervenciones y la poca explotación de los ecosistemas en estas zonas; además, es imprescindible tener en cuenta que en estas regiones aún existe una alta cobertura de bosque, también se realiza un mejor manejo y usos de las tierras y las superficies de conservación, lo que refleja un panorama con proyecciones para unas mejores condiciones de adaptación en lo referente a la biodiversidad y los servicios ecosistémicos que sirven para amortiguar los impactos esperados del cambio climático (Mapa 1).

\section{Conclusiones}

El grado de amenaza de un territorio frente a un evento climático esta directamente relacionado con la frecuencia de ocurrencia del mismo, para lo cual es necesario la revisión y análisis de los datos históricos de ocurrencia, lo que permite generar una estimación requerida para la determinación de la vulnerabilidad, en el cual además intervienen las condiciones sociales de la población representadas en las necesidades básicas insatisfechas. El

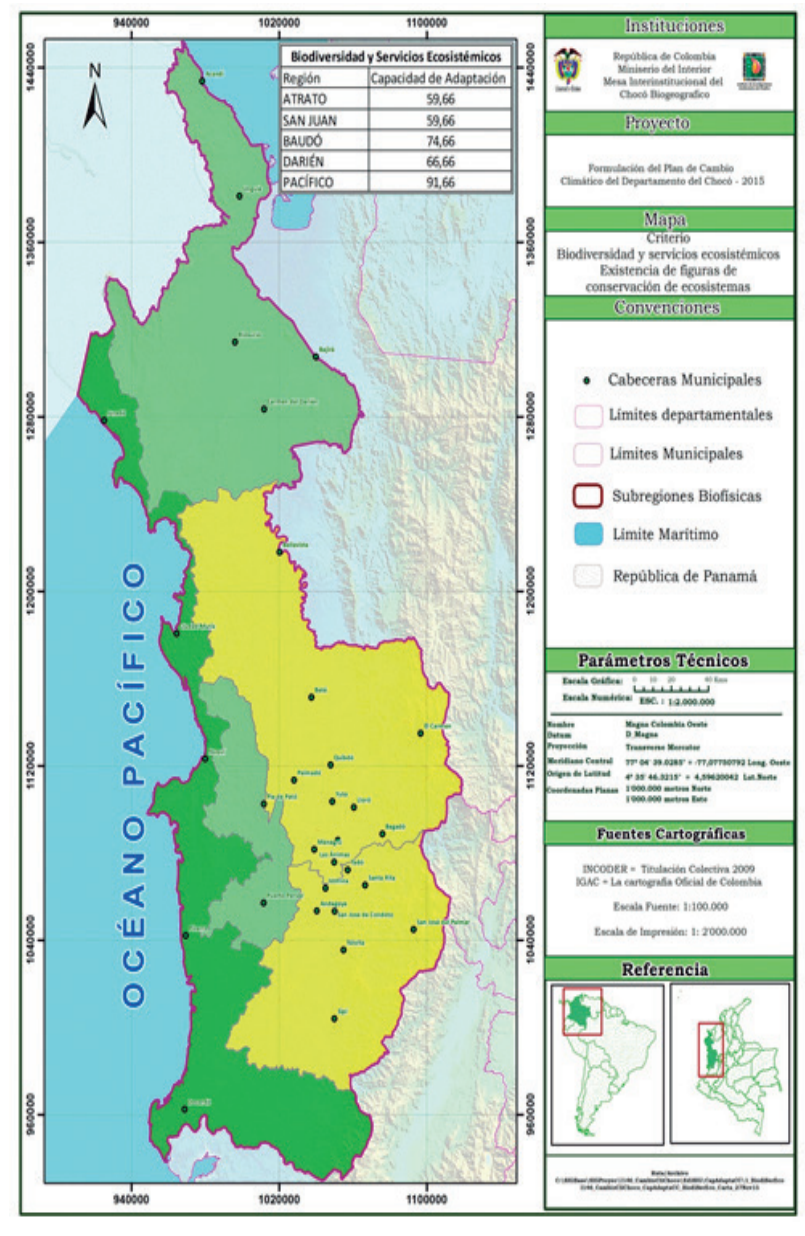

Mapa 1. Capacidad de adaptación servicios ecosistémicos

Fuente: Cartografía IGAC, Diagramado SIG- IIAP -2015

análisis se debe realizar considerando las líneas estratégicas de la política de cambio climático para lograr un análisis amplio desde las diferentes perspectivas de la situación del territorio. 
El ejercicio realizado permitió concluir que todo lo anterior es indispensable para la estimación del riesgo, donde interviene tanto la frecuencia en laocurrencia de eventos climáticos extremos como la vulnerabilidad, entendida como la interacción entre los factores ya mencionados. Asimismo, la capacidad de adaptación es resultante del disgnóstico socioambiental del área de estudio, teniendo en cuenta aspectos como la biodiversidad y servicios ecosistémicos, la gestión integral de zonas marino-costeras, insulares y oceánicas, la gestión del recurso hídrico, el estado de los sectores productivos e infraestructura básica, alimentarios y de ciudades, los cuales dan cuenta de la capacidad de respuesta de los componentes socioambientales a las amenzas que se presentan asociadas con el comportamiento del clima.

La aplicación de la metodología arrojó que la amenaza más frecuente en el departamento son las inundaciones, obteniendo los mayores grados para las subregiones de Baudó y Darién con $67 \%$, las que a su vez presentaron los mayores porcentajes de vulnerabilidad y riesgo frente a este evento climático, siendo más crítico el caso de la región Baudó. La herramienta permite que con el levantamiento de datos históricos climáticos e información ambiental y social diagnóstica, se pueda aplicar a cualquier zona del país, generando resultados para el análisis y priorización de estrategias de prevención de impactos frente al cambio climático.

\section{Literatura citada}

CORTOLIMA-UNAL, 2015. Informe final Contrato Interadministrativo $N^{\circ}$ 052-2013. Ibagué: CORTOLIMA, Universidad Nacional de Colombia, CEPREVÉ; 55 pp. URL disponible en: https://www.cortolima.gov. co/sites/default/files/images/stories/gestion_riesgos/ Gestion_del_Riesgo/Informe_Cambio_\%20Climati- co $\% 20$ cuenca $\% 20$ salda $\%$ C3\%B1a.pdf

GutiérrezE, Espinosa T. 2010. Vulnerabilidady adaptación al cambio climático. Diagnóstico inicial, avances, vacios y potenciales líneas de acción en Mesoamérica. Washington, DC: BID (Banco Interamericano de Desarrollo); 84 pp. URL disponible en: http:/www. euroclima.org/images/Publicaciones/Vulnerabilidad/ CA-BID-Vulnerabilidad_y_adaptacion_al_cambio_climatico.pdf

Instituto de Hidrología, Meteorología y Estudios Ambientales (IDEAM). (2002). Perfil del estado de los recursos naturales y del medio ambiente en Colombia 2001. Bogotá: Sistema de Información Ambiental de Colombia (SIAG); 546 pp. URL disponible en: http://documentacion.ideam.gov.co/openbiblio/bvirtual/002592/TOMO3-PERFIL.pdf

IPCC. 2012. Managing the risks of extreme events and disasters to advance climate change adaptation. Special report of the intergovernmental Panel on Climate Change. Field CB, Barros V., Stocker TF, Qin D, Dokken DJ, Ebi KL, etal. (eds.). Cambridge: Cambridge University Press, Intergovernmental Panel on Climate Change. 594 pp. URL disponible en: https://www.ipcc.ch/pdf/ special-reports/srex/SREX_Full_Report.pdf

Naciones Unidas. 1992. Convención Marco de las Naciones Unidas sobre el Cambio climático. Nueva York: NU; p. 27.

OMM, IPCC, PNUMA. (2008). Cambio climático 2007: Informe de síntesis. Contribución de los grupos de trabajo I, II y III al Cuarto Informe de evaluación del Grupo Intergubernamental de Expertos sobre el Cambio Climático. Ginebra: IPCC; 104 pp. URL disponible en: https://www.ipcc.ch/pdf/assessment-report/ar4/ syr/ar4 syr sp.pdf

PNUD. 2008. Informe sobre desarrollo humano 2007-2008. La lucha contra el cambio climático: Solidaridadfrente a un mundo dividido. New York: Programa de las Naciones Unidas para el Desarrollo (PNUD); 31 pp. URL disponible en: http://hdr.undp.org/sites/default/ files/hdr 20072008 summary spanish.pdf

PNACC. 2015. Nueva colección de escenarios de cambio climático regionalizados del Plan Nacional de Adaptación al Cambio Climático (PNACC). Datos mensuales. Bogotá: PNACC; 46 pp. URL disponible en: http:// www.aemet.es/documentos/es/serviciosclimaticos/ cambio_climat/datos_mensuales/Guia de Usuario Escenarios PNACC Datos_mensuales.pdf 